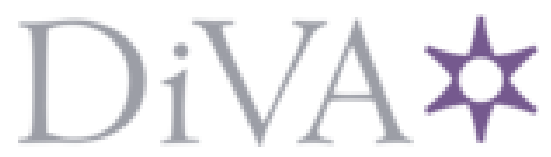

http://www.diva-portal.org

\title{
Postprint
}

This is the accepted version of a paper presented at CSCW'16 Computer Supported Cooperative Work and Social Computing San Francisco, CA, USA — February 27 - March 02, 2016.

Citation for the original published paper:

Gullström, C., Kort, J. (2016)

Where did my Office go? Is it in the Cloud!?: Workshop on Spatial and Social Connectedness in Virtual and Mediated Work Environments.

In: Darren Gergle, Meredith Ringel Morris (ed.), Proceedings of the 19th ACM Conference on Computer Supported Cooperative Work and Social Computing Companion (pp. 457-464). New York: ACM Digital Library

http://dx.doi.org/10.1145/2818052.2855525

N.B. When citing this work, cite the original published paper.

(c) Gullström, Kort | ACM 2016. This is the author's version of the work. It is posted here for your personal use. Not for redistribution. The definitive Version of Record was published in Proceedings of the ACM Conference on Computer Supported Cooperative Work, CSCW, http:// dx.doi.org/10.1145/2818052.2855525.

Permanent link to this version:

http://urn.kb.se/resolve?urn=urn:nbn:se:kth:diva- 185586 


\section{Where did my Office go? Is it in the Cloud!? - Workshop on Spatial and Social Connectedness in Virtual and Mediated Work Environments}

\section{Charlie Gullström}

KTH Royal Institute of Technology,

Department of Architecture

Stockholm, Sweden

charlieg@kth.se

Joke Kort

TNO, Human Behavior and

Organizational Innovation

Groningen, the Netherlands

Joke.kort@tno.n

ICopyrightYear $\{2016\}$

setcopyright $\{$ rightsretained $\}$

Iconferenceinfo $\{$ CSCW '16\}\{February 27 - March 02, 2016, San Francisco, CA, USA $\}$

isbn $\{978-1-4503-3950-6 / 16 / 02\}$

|doi\{http://dx.doi.org/10.1145/2818052.2855525\}

\begin{abstract}
The physical workplace, a hub for communication, collaboration and co-located interaction can no longer be taken for granted. Today, the design of intelligent interactive media, physical products and ubiquitous environments has passed the phase of being technology-driven. Meaning, insight and experience are now the key design drivers for the bridging of digital and physical design. We foresee how new interconnected knowledge systems - objects/devices, buildings and even cities created from web-based services and IoT - thoroughly transforms CSCW. A wide spectrum of services already invites users to seamlessly move between real and virtual workspaces, using a range of previously separated media channels. This interdisciplinary workshop welcomes researchers and practitioners to a day-long exchange targeting User eXperience (UX) and, specifically, the relationship between social and spatial connectedness in mediated and virtual work environments. Examples from ongoing research and developments informs a discussion on how the borders between the virtual and real become increasingly obsolete.
\end{abstract}




\section{Author Keywords}

Social, spatial presence; design; spatial, social connectedness, WebRTC; media, mediated space; audio/video communication; architecture, workplace design, interaction design

\section{ACM Classification Keywords}

H.5.m. Information interfaces and presentation (e.g. $\mathrm{HCI}$ ): Miscellaneous.

\section{Workshop theme}

In the last decade there has been numerous attempts to reach beyond the "talking heads" and the 16:9 paradigms [1] in video-mediated interaction. The most elaborate solutions have integrated heavy hardware in spaces where users benefit from how activity and social space are brought together through design, yet in very costly interiors [2]. More recently, new standards for web-based communication (WebRTC, HTML5) facilitate a representation of participants and activity space, simply via a web interface, with the help of a webcam and computer. Currently, a range of web applications for mediated interaction target technical maturation (e.g. automatic bandwidth reduction, switching microphone on/off through sound detection algorithms, adding additional tools for cooperative tasks, etc.), however, multi-party interaction, and in particular naturalness [3], and immersion and engagement, may also benefit from spatial, social and shared activity features in one seamless (virtual) space. Ongoing design research specifically addresses the fusion of spatial connectedness (a term describing how well users perceive that real space is integrated with virtual space) and social connectedness (how well users perceive social dynamics/cues and their relationship with others in mediated space during interaction) [4]. The workshop seeks to explore the seamless merging of spaces, social interaction and shared activities in virtual workspaces, in order to discuss how this may enhance the experience of (social) presence, naturalness, immersion and engagement and social connectedness.

\section{Research context}

Over twenty-five years have passed since Bill Buxton and his colleagues [5] used architectural spaces as a reference for the Hydra studies in which they showed the importance of spatial cues and design as a prerequisite for presence in shared 'person spaces' and remote collaboration. They challenged media, as a new field, to achieve what architecture as a mature discipline already does: support physical proximity by activity-based design. Activities are central to the experience of social connectedness, of engaged relationships with others as well, addressed by Goffman as Interaction rituals (recurring activities that are the building blocks of our social habits, and can have varying frequencies of occurrence) $[6,7]$ and by Clark as shared activities and grounding (social dynamics and cues needed in interaction) [8]. Today, the field of presence is arguably mature nevertheless still lacks in adequate terminology to also discuss how spatial connectedness and the experience of proximity and activity benefit and support the social connectedness. Spatial connectedness indicates that architectural design 
is as important in mediated environments as in real spaces for human interaction. In architecture, spatial features such as proximity, visibility and layout may stimulate interaction and collaboration [9]. Architectural design equally creates spatial dividers, walls and barriers by e.g. geographically separating offices or domestic environments, seeking to harbor privacy, security, but also (social) exclusion and Ioneliness [10, 11, 12]. Equally, in shared mediated spaces, the individual experience of presence is negotiated as part of a process to establish trust and confirmation from the remote party/parties [13, 14, 15]. An ongoing construction of a sense of place is informed by a series of spatial and social cues, i.e. 'reality tests' carried out by participants, who seek fast, consistent and reliable confirmation that the interaction can be trusted - in which case they can temporarily agree to suspend their judgment in favor of a 'willing suspension of disbelief'. In a broader context, social connectedness is a layered construct that describes how people relate and connect with one another in society and social groups [12]. Of relevance to the current study is that social connectedness develops over time through 'interaction rituals' $[6,7]$ (regular moments of contact and shared activities), provided that grounding (social cues) and correct social dynamics are in place [8]. The linking factor between spatial and social connectedness is the ambition to design mediated spaces that support and enhance trustbuilding processes and secure this common ground and interaction rituals.
We have identified three main developments in technology creating the challenges we meet in $\mathrm{HCI}$ design. Firstly the introduction and market penetration of low cost microcontrollers, sensors and actuators (e.g. Arduino [16]), radio beacons [17], now embedded in different products, on ourselves, in our clothing (wearables) in and outside our houses, in our cars, etc. Secondly, standardization work in network technologies and emerging industry standards (often open standards) and information exchange protocols supporting the communication of different kinds of data ranging from sensor data to real time video streams between devices, applications and services embedded in environments.

WebRTC/HTML and other new networking protocols further support easy media stream exchange and combinations of data with software applications enabling users to control features in local (as well as remote) and virtual spaces. In short, web-based service development becomes more contextual and adaptive. Thirdly, the emergence of networked systems, applications and services support data exchange, manipulation and visualisation in real time through new visualization and interaction means [4]. Progressions in the areas above express the boundaries between the physical and virtual world are dissolving. The goal is, through open development methods, to empower users to actively control and interact with and in the environment in a contextually relevant manner, allowing them to share a combination of on-site and remote uses and experiences of virtual and physical artefacts, and spaces. This workshop addresses the increasing call for designers to 
design physical equivalents of virtual artefacts (and virtual of the physical), merging the physical and the virtual even further. The new ways to interact with information, objects, people and our surroundings thus provides new challenges for $\mathrm{HCI}$ designers and calls for interdisciplinary research, new conceptual tools and methodologies to adequately design and represent content in ways that are meaningful and contribute quality of experience to users in various contexts.

\section{An example: SharedSpaces}

The SharedSpaces design prototype (Figure 1) has been developed by the workshop organisers as part of ongoing research which seeks to create a seamless mediated space for social interaction and shared activity by integrating live and/or stored media streams combined with software applications (e.g. physical sensors and actuators) that can provide live feedback and empower users to actively control features in local (as well as remote) and virtual spaces.

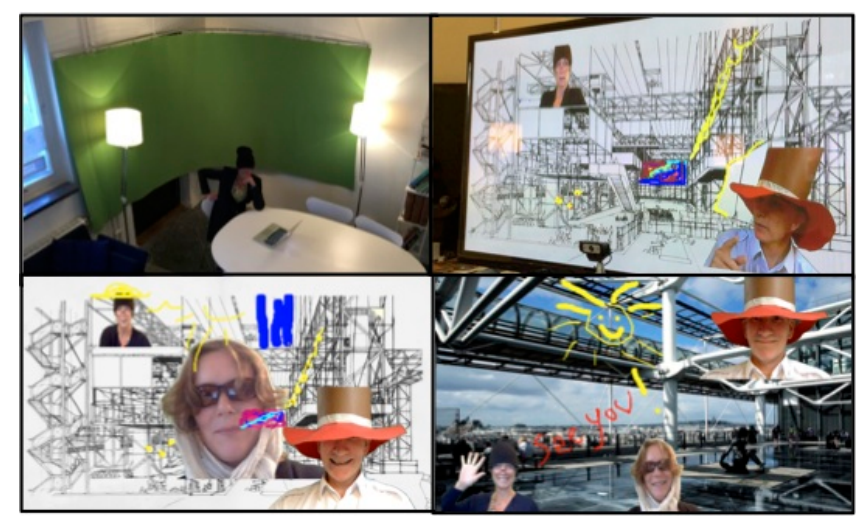

Figure 1. The SharedSpaces design prototype. Top left: An interior with green screen and participant. The other screenshots show how several participants in different locations appear side by side in SharedSpaces, see for example this YouTube movie [18].

SharedSpaces is an example of innovative WebRTC services increasingly available, inviting users to seamlessly move between real and virtual spaces using a range of previously separated media channels. It adds a spatial quality of experience by representing the users side by side in a shared virtual space. It offers a fun, novel and aesthetically appealing approach by engaging users in multiple locations to manipulate their real-time video-streams, thereby co-creating a shared space, using spatial features to fit their contextual needs. It supports social dynamics by allowing users to draw and paint together and to move and resize video streams. Further, it enhances grounding and social cues by merging video-streams and space, representing users as if they were in the same space. Standard and easily available equipment 
is used. Recent user studies show that a seamless integration of space, social dynamics and shared activity benefits the experience of presence, naturalness, immersion/engagement and social connectedness. Our ongoing design research specifically addresses the fusion of spatial connectedness (a term describing how well users perceive that real space is integrated with virtual space) and social connectedness (how well users perceive social dynamics/cues and their relationship with others in mediated space during interaction).

\section{Workshop goals}

We find that the research field today lacks in adequate terminology to discuss how spatial connectedness and the experience of proximity and activity benefit and support social connectedness in mediated and virtual work collaboration. Spatial connectedness indicates that architectural design is as important in mediated environments as in real spaces for human interaction. In architecture, spatial features such as proximity, visibility and layout may stimulate interaction and collaboration [9]. Architectural design equally creates spatial dividers, walls and barriers by e.g. geographically separating offices or domestic environments, seeking to harbour privacy, security, but also (social) exclusion and loneliness [10, 11, 12] Equally, in shared mediated spaces, the individual experience of presence is negotiated as part of a process to establish trust and confirmation from the remote party/parties [13, 14, 15]. An ongoing construction of a sense of place is informed by a series of spatial and social cues, i.e. 'reality tests' carried out by participants, who seek fast, consistent and reliable confirmation that the interaction can be trusted and understood [8] - in which case they can temporarily agree to suspend their judgment in favour of a 'willing suspension of disbelief'. In a broader context, social connectedness is a layered construct that describes how people relate and connect with one another in society and social groups [12]. Of relevance is that social connectedness develops over time through 'interaction rituals' (regular moments of contact and shared activities), provided that grounding (social cues) and correct social dynamics are in place. The linking factor between spatial and social connectedness is the ambition to design mediated spaces that support and enhance trust-building processes and secure this common ground and interaction rituals. The above results in new challenges and opportunities for $\mathrm{HCI}$ researchers with interests ranging from direct and live representations in virtual space (not least facilitated by new visualization and interaction techniques) to subtler interaction that supports an awareness of persons' or objects' remote (inter)actions.

In the workshop we seek to explore from a user experience perspective what the important issues are in designing and evaluating these merged environments and new interactions. Specifically, we seek contributions that address theory, methodology and practice (case studies) in the areas of: 1) the design of spaces (physical, virtual and mixed with sensor and actuator technology), how the design of such spaces is supporting and merged with 2) interactions with objects, others and other spaces and the intended goals with these interactions in terms of UX, 3) case studies providing insight in the created UX and 4) what technical implementations are used to realize this. We are specifically interested in applications of mainstream technology because these often better support in-situ 
and long term studies from which we can learn most in terms of UX and the value provided in our everyday work. For example, our own work focuses on spatial and social connectedness design in mediated communication that seamlessly supports merging of spaces, objects therein, people and activities performed in such mediated communication environments. UX concepts such as spatial and social connectedness are key experiences which we hypothesize creates value in terms of overall well-being, relatedness, identity, social support, and more control over work events.

\section{Workshop activities}

This one-day workshop seeks to attract 20-25 participants. Position papers (max 4 pages) are accepted from a wide range of disciplines, e.g. interaction researcher and designers, architects, WebRTC-developers, IoT service innovators.

Participants are asked to share experiences, user studies and evaluations from collaborative practices that relate to the following: seamless integration of space, social dynamics and shared activity (in virtual space facilitated real-time communication standards (e.g. WebRTC), advanced sensor technology, visualization and interaction techniques, alongside other tools approaching the market). Means used for recruiting and selecting participants are a call for participation distributed through different ACM CHI mailing lists, social media and the professional networks of the authors and their colleagues (e.g. LinkedIn). A workshop website will provide further information on the call for participation, the workshop goals, context, accepted position papers, the workshop program and different means for communication among the organizers, participants and interested public at large.
Preliminary workshop agenda:

8.30-9.30 Coffee and informal preparations for the workshop -9.30-10.00 Introduction and round of presentations - 10-11.30 Parallel work sessions in two groups • 11.30-12 Reporting back • 12.00-13.00 Lunch -13.00-14.00 A session to identify relevant futureoriented topics, based on the morning's work $\bullet 14.00-$ 16.00 Parallel discussion groups explore 4-5 selected topics for a 'Draft Agenda' (including coffee) • 16.0017.00 Concluding session with a round table to comment the Draft Agenda and propose future steps • 17.00-18.00 A walk or other social activity • 19.00 Dinner

Workshop planning:

As soon as the workshop has been accepted, the organisers will plan short weekly video-meetings to prepare and divide the organisational work. All papers that are submitted will be read and commented on by a reviewing committee selected, invited and coordinated by the workshop organizers.

Participants who are interested in this workshop are requested to: (1) Submit a short paper (4 pages) addressing a relevant topic in relation to the call; (2) Participate in a video-meeting with the workshop organisers, if the paper is accepted for workshop participation; (3) Upload additional information on the workshop website (e.g. photos, videos, links, other publications or design experiences). (4) Partake in two preparatory video-meetings for planning and community building, where brief presentations by all participants will ensure that everyone is acquainted with everyone's work. 
After the workshop, the organisers will summarize the outcome and formulate a Post-Workshop Statement and Invitation to continued collaboration, which provides an outline of the topics or issues for a potential co-authored article, based on the outcome of the workshop. Within a month after the workshop, the organisers will then invite all interested participants to continue discussions in view of preparing a co-authored paper, based on the insights that the workshop has generated. It is expected that a majority of participants, although not all, will want to continue the collaboration. In this phase, the organisers will take the role as editors asking the participants to contribute as follows: (1) Participate in at least two of six mediated mini-seminars that the organisers will host in the SharedSpaces virtual meeting space, addressing different topics; (2) Write draft texts sections and submit relevant references, following interests and expertise; (3) Volunteer to proof-read and provide feedback at later stages of the co-authoring process.

The editorial group will use own resources to edit the article and a draft version of the article is planned to be ready for submission by September 2016.

\section{Organizers background}

Charlie Gullström, PhD, Architect SAR/MSA, is a University Lecturer at the department of Architecture (KTH Royal Institute of Technology, Stockholm), where she combines teaching with research and heads the research group KTH Smart Spaces: Architecture and Interactive Media. Her design-driven research and practice over twenty years address the fusion of architecture, interaction design and media technology, facilitated by new information and communication technology. Her particular interest concerns the contribution from architects to a highly-mediated society, given that new digital tools have thoroughly changed the way humans interact and communicate and thus may trigger more sustainable patterns of behaviour: the design of responsive environments can provide live feedback to users (sustainable design). Gullström initiated the VINNOVA Centre of Excellence for Sustainable Communications at KTH and served as its Centre Director 2005-2007. She is currently research leader for a 3-year EU-project

(www.compeit.eu) which develops web-based services for highly interactive shared media experiences.

Joke Kort has a degree in cognitive psychology and social informatics from the State University of Groningen and a Master in Technological Design from the Stan Ackermans Institute, Eindhoven University of Technology. She works for TNO on multidisciplinary client and research projects in different domains such as education, healthcare, telecommunications, work environments, public services and domestic applications. Her work focuses on user experience in these domains, what value technology offers to its users, and its effects on behaviour on a personal as well as societal level. She held a 6 months postdoc position at the Delft University of Technology, Electrical Engineering, Mathematics and Computer Science and is a guest researcher at this university in the faculty of Industrial Design Engineering. Currently she holds a part time position as senior invited researcher at KTH in the department of Architecture.

\section{Acknowledgements}

The workshop is organized with acknowledgments to the EU FP7 COMPEIT project (www.compeit.eu). COMPEIT explores the future of connected media and 
creates a web-based system for highly interactive, personalized, shared media experiences. The project is funded by the European Union.

\section{References}

1. Joke Kort, Charlie Gullstrom, Harold C. Nefs. 2013. Beyond talking heads - Towards sharing life. In the Proceedings of the SIGCHI Conference on Human Factors in Computing Systems (CHI'13) workshop The Future of Personal Video Communication: Moving Beyond Talking Heads to Shared Experiences.

2. Cisco Immersive Telepresence. Retrieved September 24, 2015 from

http://www.ivci.com/products/cisco/ciscoimmersive-telepresence/

3. Ned Kock. 2002. Evolution and Media Naturalness: A look at E-communication through a Darwinian Theoretical Lens. In Proceedings of the TwentyThird International Conference on Information Systems (ICIS'02).

4. Joke Kort, Charlie Gullstrom, Leif Handberg, Galena Kostoska, Sylvia van der Pal. 2016. CHI2016 Note, submitted and currently in review.

5. Bill W. Buxton. 1992. Telepresence: integrating shared task and person spaces. In the Proceedings of Graphics Interface '92, 123-129.

6. Erving Goffman. 1961. The presentation of self in everyday life. New York, Doubleday.

7. Erving Goffman. 1967. Interaction ritual: Essays on face-to-face behaviour. Garden City, NY Doubleday.

8. Herbert H. Clark. 1996. Using language. Cambridge University Press.

9. Hillier, B. 1996. Space is the Machine: A Configurational Theory (Cambridge: Cambridge University Press)
10. John T. Cacioppo and William Patrick. Loneliness: Human Nature and the Need for Social Connection. 2009. W. W. Norton \& Company.

11. Marco C. Rozendaal and Nienke Hollenberg. 2013. Pediatric Oncology Sketched Out. TU Delft, Delft.

12. Sabine Wildevuur, Dick van Dijk, Anne Äyväri, Mie Bjerre, Thomas Hammer-Jakobsen, and Jesper Lund. 2013. Connect - Design for an Emphatic Society. BIS Publishers.

13. Charlie Gullstrom (Charlie add)

14. Wijnand A. IJsselsteijn (2004). Presence in depth. Doctoral dissertation. Eindhoven: Eindhoven University of Technology

15. Wijnand A. IJsselsteijn, Riva, G. (2003). Being there: The experience of presence in mediated environments', In: G. Riva, F. Davide, \& W.A. IJsselsteijn (Eds.) Being there: Concepts, effects and measurement of user presence in synthetic environments. Amsterdam: IOS Press

16. https://www.arduino.cc/

17. http://estimate.com and https://google.github.io/physical-web/

18. SharedSpaces YouTube movie: https://www.youtube.com/watch?t=13\&v=lsZYk4T mI14 